\title{
Thermodynamic Control over Molecular Aggregate Assembly Enables Tunable Excitonic Properties across the Visible and Near-Infrared \\ Supporting Information
}

Arundhati P. Deshmukh, ${ }^{1}$ Austin D. Bailey, ${ }^{1}$ Leandra S. Forte, ${ }^{1}$ Xingyu Shen, ${ }^{1,2}$ Niklas Geue, ${ }^{1,3}$ Ellen M. Sletten ${ }^{1}$ and Justin R. Caram ${ }^{1 *}$

${ }^{1}$ Department of Chemistry and Biochemistry, University of California, Los Angeles, 607 Charles E. Young Dr. East, Los Angeles, California-90095, United States. ${ }^{2}$ College of Chemistry and Molecular Engineering, Peking University, Beijing 100871, P.R.China. ${ }^{3}$ Department of Chemistry and Mineralogy, Leipzig University, Johannisallee 29, 04103 Leipzig, Germany.

*Corresponding author

Email: jcaram@chem.ucla.edu 


\section{Table of Contents}

1. Characterization of aggregates

2. Three component equilibrium model

3. Supporting results from the model

4. Non-negative matrix factorization

5. Obtaining experimental Gibbs free energies

6. Applying design principles to TDBC

7. Experimental section 


\section{Characterization of aggregates}

\subsection{Diffusion ordered spectroscopy (DOSY)}
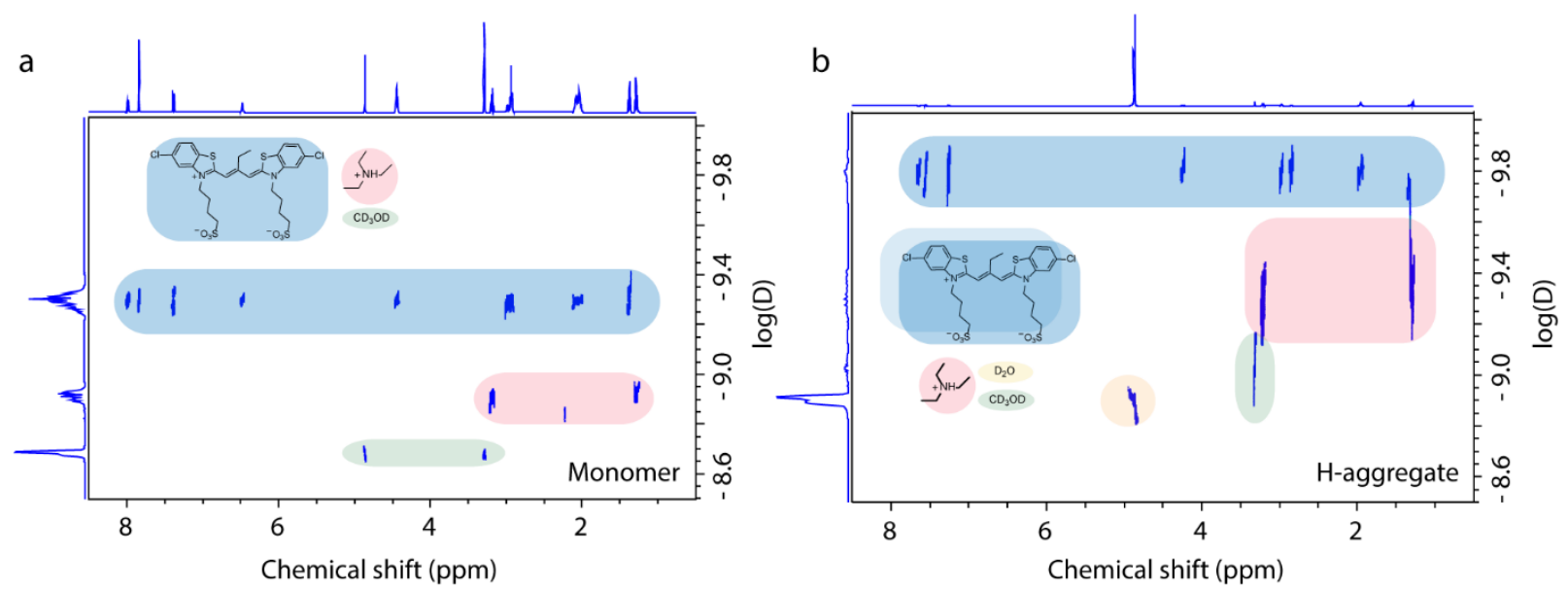

Figure S1: Comparison of DOSY spectra of a. Cy3-Et monomer $(5 \mathrm{mM})$ in $\mathrm{CD}_{3} \mathrm{OD}$ and b. Cy3-Et Haggregate $(1 \mathrm{mM})$ in $10 \% \mathrm{CD}_{3} \mathrm{OD} / \mathrm{D}_{2} \mathrm{O}(\mathrm{v} / \mathrm{v})$.

Figure S1a shows the DOSY spectrum of Cy3-Et monomer. Similar to the H-aggregates DOSY from main text (also shown in Figure S1b on same scale as the monomer for comparison), all the protons from the dye molecules show up at a unique diffusion constant. We use Stokes-Einstein relationship to estimate the size from the diffusion constant. For viscosity of solvent the mixture, a weighted average of the known viscosities for $\mathrm{D}_{2} \mathrm{O}$ and $\mathrm{CD}_{3} \mathrm{OD}$ was used. The hydrodynamic radii and other calculation parameters, given in Table S1, show that the $\mathrm{H}$-aggregate is roughly twice the size of the monomer.

Table S1. Diffusion coefficients obtained from DOSY and parameters used to calculate the hydrodynamic radii of $\mathrm{Cy} 3$-Et monomers and $\mathrm{H}$-aggregates from Stokes-Einstein relation.

\begin{tabular}{lclcc}
\hline Sample & Diffusion Coefficient $\left(\mathbf{m}^{2} \mathbf{s}^{-1}\right)$ & Solvent & Viscosity $\left(\mathbf{k g m}^{-1} \mathbf{s}^{-1}\right)$ & $\begin{array}{l}\text { Hydrodynamic } \\
\text { Radius (nm) }\end{array}$ \\
\hline H-agggregate & $1.32 \times 10^{-10}$ & $10 \% \mathrm{CD}_{3} \mathrm{OD} / \mathrm{D}_{2} \mathrm{O}$ & $1.05 \times 10^{-3}$ & 1.56 \\
$(1 \mathrm{mM})$ & $4.96 \times 10^{-10}$ & $100 \% \mathrm{CD}_{3} \mathrm{OD}$ & $6.0 \times 10^{-4}$ & 0.72 \\
Monomer $(5 \mathrm{mM})$ & & & \\
\hline
\end{tabular}

\subsection{Atomic Force Microscopy of J-aggregate}

We use AFM analysis to probe the height of 2D sheets and their layer structure. As Cy3-Et J-aggregate solutions require added salt, complicating height analysis, Cy7-Ph J-aggregates (prepared without salt at $20 \% \mathrm{MeOH}, 0.2 \mathrm{mM}$ dye as described in our prior work) were analyzed. ${ }^{1}$ AFM images of Cy7-Ph (main text Figure $2 \mathrm{c}$ ) are fit to the difference in heights between adjacent layers. ${ }^{2}$ We plot a histogram of the heights of a selected region (Figure $2 \mathrm{c}$ bottom inset) and fit to a sum of 3 Gaussians, $y=\sum_{i=1}^{3} \mathrm{a}_{\mathrm{i}}$. $\mathrm{e}^{-\left(\frac{x-b_{i}}{c_{i}}\right)^{2}}$ where $a_{i}$, and $b_{i}$ respectively are the amplitudes, center positions of the Gaussians and $c_{i}$ are 
related to width. The height of a single sheet was calculated from the difference in the center positions of adjacent peaks $\left(b_{i+1}-b_{i}\right)$, and is $1.18 \pm 0.01 \mathrm{~nm}$, which matches the height of a Cy7-Ph molecule 1.2 $\mathrm{nm}$ (Figure 2c, top inset). Thus, we conclude that the sheets are monolayers with the molecular plane perpendicular to the plane of the sheets. Since we do not observe significant differences in spectral lineshapes or electron microscope images for different dyes studied here, we hypothesize that this aggregate morphology applies to all J-aggregates studied.

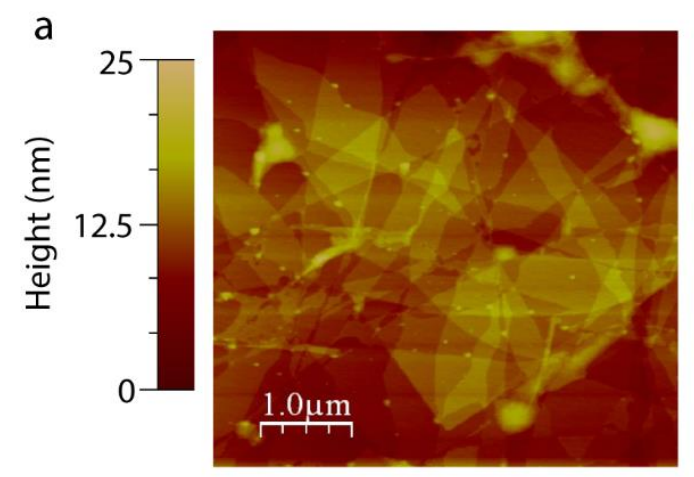

Figure S2. AFM image of a drop casted film of Cy7-Ph on freshly cleaved mica with a wider view showing sheet morphology. Height analysis confirms the sheets are monolayers.

\subsection{Aggregate spectra over long time}
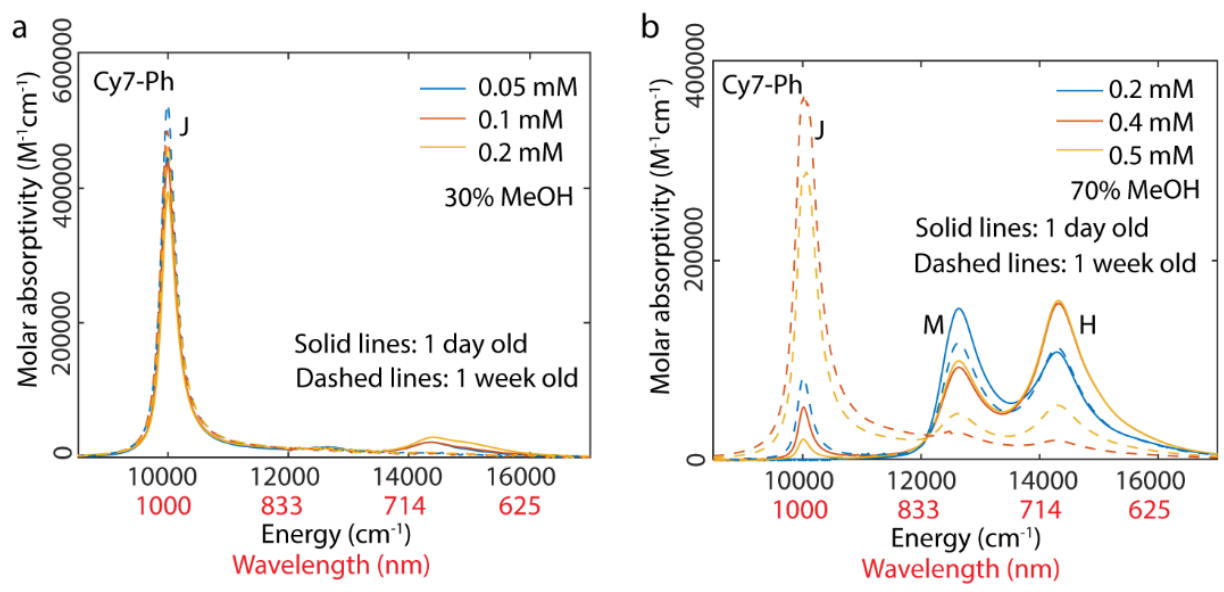

Figure S3. Absoprtion spectra of Cy7-Ph aggregates; a. $30 \% \mathrm{MeOH}$, b. $70 \% \mathrm{MeOH}$ at different concentrations taken $24 \mathrm{hrs}$ after sample preparation (solid lines) and after 1 week of sample preparation (dashed lines).

\section{Three component equilibrium model}

\subsection{Characteristic Polynomial Derivation}

We model the equilibrium between the monomer, $\mathrm{H}$-aggregate and J-aggregate using the following equations:

$$
n_{1} M \rightleftharpoons H
$$




$$
n_{2} H \rightleftharpoons J
$$

where $M, H$, and $J$ denote the monomer, $\mathrm{H}$-aggregate and J-aggregate respectively. $n_{1}$ denotes the number of monomers in an $\mathrm{H}$-aggregate and $n_{1} \cdot n_{2}$ is the total number of monomers in the J-aggregate. Initially, the monomers assemble into an $\mathrm{H}$-aggregate, which is a dimer. When the total number of monomers reach a certain limit, they assemble into a large J-aggregate, which is a 2D sheet in all the cases discussed here. This assumption is justified by our experimental observations where we see an $\mathrm{H}$ aggregate peak rising initially, which then goes down with a sudden increase in the J-aggregate peak. The equilibrium constant is related to standard Gibbs free energy by $\Delta G^{o}=-R T \ln (K)$, where $\mathrm{R}, \mathrm{T}$ and $\mathrm{K}$ are the universal gas constant, standard temperature and equilibrium constant respectively.

Let $K_{1}$ and $K_{2}$ denote the equilibrium constants for equations (1) and (2) respectively. Therefore,

$$
\begin{gathered}
K_{1}=\frac{C_{H}}{C_{M}^{n_{1}}} \\
K_{2}=\frac{C_{J}}{C_{H}^{n_{2}}}
\end{gathered}
$$

Equation (3) can be rearranged to

$$
C_{H}=K_{1} \cdot C_{M}^{n_{1}}
$$

Substituting eq. (5) into eq. (4) and rearranging eq. (4),

$$
C_{J}=K_{2} \cdot\left(K_{1} \cdot C_{M}^{n_{1}}\right)^{n_{2}}=K_{1}^{n_{2}} \cdot K_{2} \cdot C_{M}^{n_{1} \cdot n_{2}}
$$

Further, if $C_{\text {tot }}$ is the total dye concentration in the solution,

$$
C_{t o t}=C_{M}+n_{1} \cdot C_{H}+n_{1} \cdot n_{2} \cdot C_{J}
$$

Substituting eq. (5) for $C_{H}$ and eq. (6) for $C_{J}$ into eq. (7),

$$
n_{1} \cdot n_{2} \cdot K_{2} \cdot K_{1}^{n_{2}} \cdot C_{M}^{n_{1} \cdot n_{2}}+n_{1} \cdot K_{1} \cdot C_{M}^{n_{1}}+C_{M}-C_{\text {tot }}=0
$$

or

$n_{1} \cdot n_{2} \cdot e^{-\Delta G_{2}^{o} / R T} \cdot e^{-n_{2} \cdot \Delta G_{1}^{o} / R T} \cdot C_{M}^{n_{1} \cdot n_{2}}+n_{1} \cdot e^{-\Delta G_{1}^{o} / R T} \cdot C_{M}^{n_{1}}+C_{M}-C_{t o t}=0$

Eq. (8) can be solved analytically for $C_{M}$ for a known value of $C_{t o t}$. $C_{H}$ and $C_{J}$ were obtained using eq. (5) and eq. (7) respectively. We get the mole fractions $(\chi)$ of each component using eq. (9).

$$
\begin{aligned}
& \chi_{M}=C_{M} / C_{t o t} \\
& \chi_{H}=\left(n_{1} \cdot C_{H}\right) / C_{t o t} \\
& \chi_{J}=\left(n_{1} \cdot n_{2} \cdot C_{J}\right) / C_{t o t}
\end{aligned}
$$

All mole fraction curves are calculated by solving the above polynomial, (8) using standard root finding functions in MATLAB (code available upon request) for a set of parameters $n_{1}, n_{2}, \Delta G_{1}^{o}$ and $\Delta G_{2}^{o}$ while varying $C_{t o t}$. The aggregation map shown in main text Figure $3 \mathrm{a}-\mathrm{c}$ is calculated by fixing $n_{1}, n_{2}, C_{t o t}$ while varying $\Delta G_{1}^{o}$ and $\Delta G_{2}^{o}$. The obtained mole fractions $\chi_{M}, \chi_{H}, \chi_{J}$ were used as RGB values for each pixel on 
the maps in Figures 3a-c with red, green and blue corresponding to J-aggregate, monomer and $\mathrm{H}$ aggregate respectively.

\subsection{Number of real positive roots of the polynomial:}

For $n_{1}=2$, (8) can be simplified to a general form $a x^{m}+b x^{2}+x-c=0$ where $m$ is a large even number and $a, b, c>0$. It can be proven that any general equation of this form will only have one real positive root.

Let us denote the polynomial as $P(x)=a x^{m}+b x^{2}+x-c$. Therefore, $P(0)=-c$ and $\lim _{x \rightarrow \infty} P(x)=\infty$. Using intermediate value theorem, $P(x)$ will have at least one real positive root. Further, $P^{\prime}(x)=\max ^{m-1}+2 b x+1$ which is always positive for all $x \geq 0$ as long as $a, b, c>0$ which is true in this case (equilibrium constants and aggregation numbers are always positive). In other words, $P(x)$ is a monotonically increasing function for all $x>0$. Using Rolle's theorem, $P(x)$ can have at most one real positive root. Therefore, $P(x)$ has exactly one real positive root.

\subsection{Dependence on choice of pathway}

This model does not depend on the choice of pathway. We obtain the same characteristic polynomial if we write the equilibrium equations as monomers adding on to $\mathrm{H}$-aggregates instead of $\mathrm{H}$-dimers exclusively reacting to form J-aggregates, as shown below.

$$
H+\left(n_{1} \cdot n_{2}-n_{1}\right) M \rightleftharpoons J
$$

Here, we write eq. (2) as monomers adding on to the $\mathrm{H}$-aggregate as opposed to $\mathrm{H}$-aggregates combining to form a J-aggregates. The stoichiometric coefficient $n_{1} \cdot n_{2}-n_{1}$ is necessary to balance the equation. Let $K_{2}^{\prime}$ denote the equilibrium constant for eq. (10).

$$
K_{2}^{\prime}=\frac{C_{J}}{C_{H} \cdot C_{M}^{n_{1} \cdot n_{2}-n_{1}}}
$$

Substituting $C_{H}$ from eq. (5) and rearranging,

$$
C_{J}=K_{2}^{\prime} \cdot K_{1} \cdot C_{M}^{n_{1} \cdot n_{2}}
$$

In order to relate the $K_{2}^{\prime}$ with $K_{2}$, we multiply eq. (1) by $n_{2}-1$ and add to eq. (2), resulting in eq. (10).

$$
\begin{array}{llc} 
& \left(n_{2}-1\right) \cdot n_{1} \cdot M \rightleftharpoons\left(n_{2}-1\right) H ; & K_{1}^{n_{2}-1} \\
+\quad & n_{2} H \rightleftharpoons J ; & K_{2} \\
\hline H+\left(n_{1} \cdot n_{2}-n_{1}\right) M \rightleftharpoons J ; & K_{1}^{n_{2}-1} \cdot K_{2}
\end{array}
$$

Therefore, we get $K_{2}^{\prime}=K_{1}^{n_{2}-1} \cdot K_{2}$. Substituting this in eq. (12), we obtain a relationship that is same as eq. (6),

$$
C_{J}=K_{1}^{n_{2}} \cdot K_{2} \cdot C_{M}^{n_{1} \cdot n_{2}}
$$

Thus, substituting eq. (5) and (12) for $C_{H}$ and $C_{J}$ respectively, in eq. (7), we obtain the same characteristic polynomial as eq. (8).

$$
n_{1} \cdot n_{2} \cdot K_{2} \cdot K_{1}^{n_{2}} \cdot C_{M}^{n_{1} \cdot n_{2}}+n_{1} \cdot K_{1} \cdot C_{M}^{n_{1}}+C_{M}-C_{t o t}=0
$$




\section{Supporting results from the model}

\subsection{Effect of aggregation number}

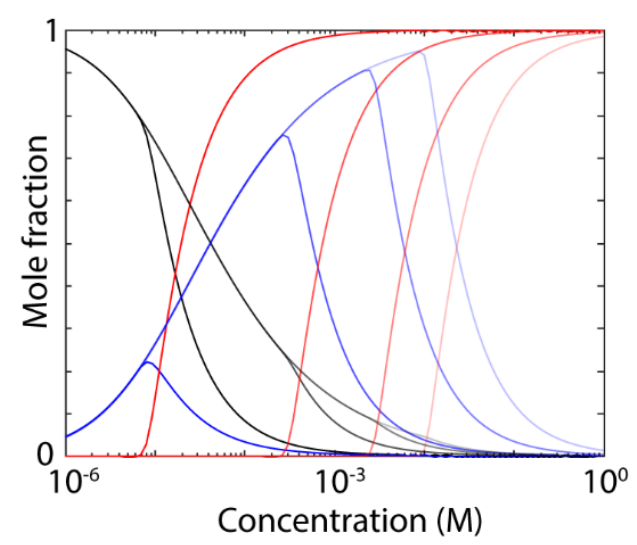

Figure S4. Mole fractions of the monomer (black), H-aggregates (blue) and J-aggregates (red) as a function of total dye concentration for $\Delta \mathrm{G}_{1}{ }^{\circ}=-25 \mathrm{~kJ} / \mathrm{mol}$ and $\Delta \mathrm{G}_{2}{ }^{\circ}=-300 \mathrm{~kJ} / \mathrm{mol} ; \mathrm{n}_{1}=2$ and $\mathrm{n}_{2}=10,15$, 20 and 25 (darkest to lightest respectively).

Given the extended nature of J-aggregates, realistic $\mathrm{n}_{2}$ values to be used in the model should be very large $\left(>10^{2}\right)$. However, finding roots of high order polynomials with existing algorithms is subject to error. To assess the approximation of the effect of increasing aggregation number in (8), we plot mole fraction in Figure S4 for higher $n_{2}$ values. With increasing $n_{2}$ we observe a delayed onset or sharp 'turn-on' of Jaggregation concurrent with a sharp decrease in monomer and $\mathrm{H}$-aggregate.

\section{Non-negative matrix factorization}

We obtain the mole fraction from experimental data using non-negative matrix factorization (NNMF). In NNMF, a large matrix $\mathbf{V}$ is factorized into two matrices $\mathbf{W}$ and $\mathbf{H}$ where all elements of the matrices are non-negative numbers. We construct a matrix of the spectra shown in Figure S5 with each row representing a spectrum at a different dye concentration, $\mathbf{V}_{\mathrm{m} \times \mathrm{n}}$ where $\mathrm{m}$ and $\mathrm{n}$ are the number of samples and number of elements in one spectrum respectively. Using the standard NNMF package from MATLAB, we factorize this matrix into a weights matrix $\mathbf{W}_{\mathrm{m} \times 3}$ and a spectra matrix $\mathbf{H}_{3 \times n}$ where each row represents the spectrum of a pure component. We narrow down the NNMF algorithm by using starting points for known spectra of monomers and J-aggregates. While pure monomer spectrum is easily isolated, we chose a spectrum at high dye and salt concentration at low $\% \mathrm{MeOH}$ for the J-aggregate since these conditions yield mostly J-aggregated species.

$\boldsymbol{V}_{m \times n}=\boldsymbol{W}_{m \times 3} * \boldsymbol{H}_{3 \times n}$

The factors $\boldsymbol{W}$ and $\boldsymbol{H}$ are chosen such as to minimize the root mean square residual $(F)$ of the product with original matrix where $F=\sqrt{\frac{\sum n, m|\boldsymbol{V}-\boldsymbol{W} * \boldsymbol{H}|^{2}}{n * m}}$. The matrix $\boldsymbol{H}$ is plotted in main text Figure $3 \mathrm{~g}$. The three vectors obtained from NNMF represent the spectra of each component i.e. monomer, $\mathrm{H}$-aggregate and Jaggregate. We normalize the weights matrix $\boldsymbol{W}$ along each row to get experimental mole fractions (circles on main text Figure $3 \mathrm{~h}) W_{i j}=\frac{W_{i j}}{\sum_{j} W_{i j}}$. We interpolate this function and obtain the crossing point where 
the normalized weights corresponding to the monomer and $\mathrm{H}$-aggregate are equal. Later on, we will use eq. (17) to obtain the experimental value of $\Delta G_{1}^{o}$ from the monomer $\mathrm{H}$-aggregate crossing concentration.

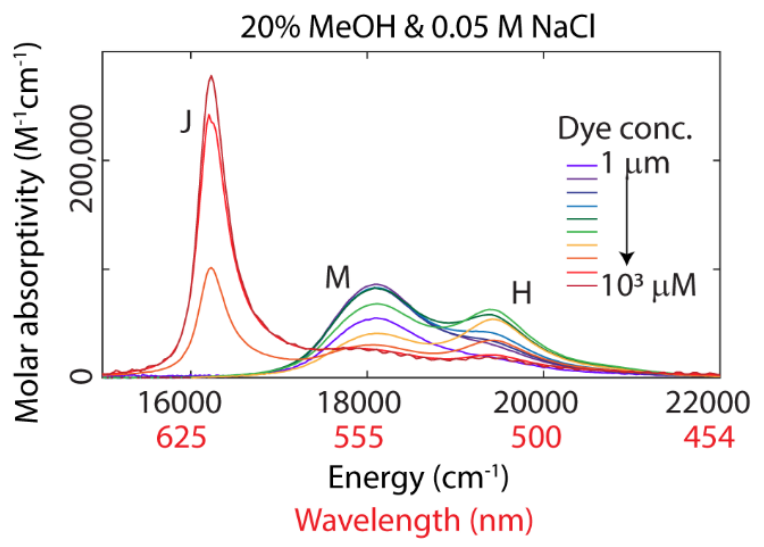

Figure S5. Experimental concentration dependence spectra of Cy3-Et in $20 \% \mathrm{MeOH}$ and $0.05 \mathrm{M}$ salt. This dataset was used to perform NNMF.

\section{Obtaining experimental Gibbs free energies}

Derivation of analytical expression for $\Delta G_{1}^{o}$ : To decrease the fitting space, we derive a simple analytical expression for $\Delta G_{1}^{o}$ using the monomer to $\mathrm{H}$-aggregate crossing point. We apply the condition $\chi_{M}=\chi_{H}$ or $C_{M}=n_{1} \cdot C_{H}$ on eq. (8). For $n_{1}=2$, combining this condition with equation (3) gives $C_{M}=1 /\left(2 \cdot K_{1}\right)$. Substituting this in eq. (8), we get

$$
\begin{gathered}
\frac{n_{2} \cdot K_{2}}{2^{\left(2 n_{2}-1\right)}} \cdot\left(\frac{1}{K_{1}}\right)^{n_{2}}+\frac{1}{K_{1}}-C^{\prime}{ }_{\text {tot }}=0 \\
\frac{n_{2} \cdot e^{-\Delta G_{2}^{o} / R T}}{2^{\left(2 n_{2}-1\right)}} \cdot e^{\frac{n_{2} \cdot \Delta G_{1}^{o}}{R T}}+e^{\frac{\Delta G_{1}^{o}}{R T}}-C^{\prime}{ }_{\text {tot }}=0
\end{gathered}
$$

where $C^{\prime}$ tot is the dye concentration where monomer and $\mathrm{H}$-aggregate mole fractions are same and $\frac{1}{K_{1}}=$ $e^{\Delta G_{1}^{o} / R T}$. For $\Delta G_{1}^{o}<0$, taking the limit $n_{2} \rightarrow \infty$, eq. (15) can be reduced to

$\frac{1}{K_{1}}=C_{t o t}$ or $e^{\Delta G_{1}^{o} / R T}=C^{\prime}{ }_{t o t}$

Therefore, $\Delta G_{1}^{o}=R T \ln \left(C^{\prime}{ }_{\text {tot }}\right)$

Interpolating the experimental mole fractions obtained from NNMF in main text Figure $3 \mathrm{~h}$, we obtain $C^{\prime}{ }_{\text {tot }}=40.8 \mu M$ which results in $\Delta G_{1}^{\circ}=-25.0 \mathrm{~kJ} / \mathrm{mol}$.

Fitting model to experimental data: We define a custom function using the polynomial in (8) with $n_{1}, n_{2}$ as fixed parameters and $\Delta G_{1}^{o}, \Delta G_{2}^{o}$ free parameters. We use the analytically obtained value of $\Delta G_{1}^{o}$ as a starting point in the fitting. Narrowing down the possible range of $\Delta G_{2}^{o}$ value, on the other hand, was more complicated due to its strong dependence on $n_{2}$. Therefore, we fix the aggregation numbers in our model to $n_{1}=2$ and $n_{2}=30$ (limited by our computational capacity) while fitting the data in main text Figure $3 \mathrm{~h}$. We get an estimate of $\Delta G_{2}^{o}$ by visual inspection of the mole fraction curves and then use this estimate as a starting point in the least squares curve fitting. We then fit our three component model to this data, finding good agreement for $n_{1}=2$ and $n_{2}=30$ (greater values of $n_{2}$ are numerically unstable) 
and optimize the values of $\Delta \mathrm{G}_{1}^{\circ}=-24.8 \mathrm{~kJ} / \mathrm{mol}, \Delta \mathrm{G}_{2}^{\circ}=-740.2 \mathrm{~kJ} / \mathrm{mol}$ using least-squares curve fitting (lines in Figure 3h).

\section{Applying design principles to TDBC}

Figure $\mathrm{S} 6$ self-assembly of a benzimidazole cyanine dye TDBC. We are able to tune the self-assembly from monomer to J-aggregate state by independently tuning the dye concentration or solvent to non-solvent ration $(\% \mathrm{MeOH})$ while keeping the other factor constant. As opposed to rest of the benzothiazole dyes studied here, TDBC monomers do not go through an $\mathrm{H}$-aggregated dimer or oligomer before forming the extended J-aggregates structure. TDBC still follows similar general trends where lower $\% \mathrm{MeOH}$ and higher dye concentrations favor the formation of extended J-aggregates which are also found to be 2D sheets.
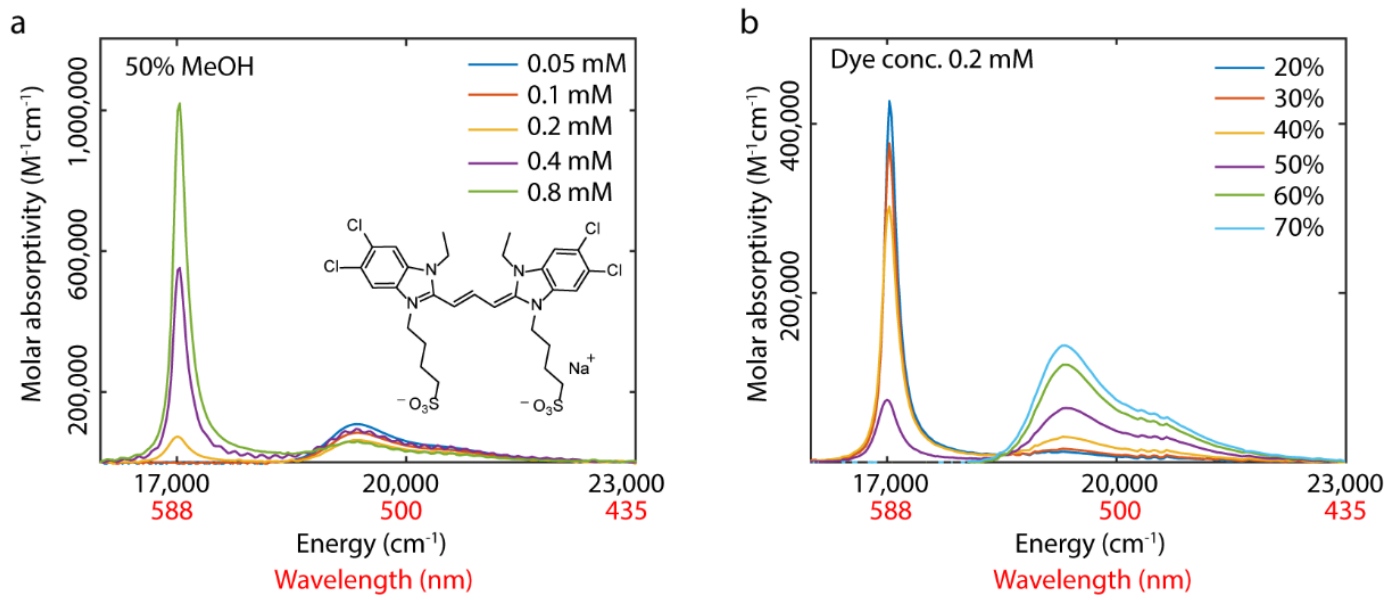

Figure S6. Absorption spectra of TDBC dye aggregates formed by independently tuning a. dye concentration in MeOH:water mixtures with $50 \% \mathrm{MeOH}$ v/v; (inset) structure of TDBC dye; b. $20 \%$ $70 \% \mathrm{MeOH}$ in methanol-water mixtures with fixed dye concentration of $0.2 \mathrm{mM}$.

\section{Experimental Section}

Materials: All dyes were obtained from FEW chemicals GmbH (catalog \# S0046, S2278, S2284, S2433 and S0837 for TDBC, Cy3-Et, Cy5-Ph, Cy7-Ph and Cy7-DPA respectively). Sodium chloride, sucrose, D-(+)trehalose and methanol were obtained from Thermo Fisher Scientific. Milli-Q water (18 $\mathrm{M} \Omega$ ) was used for making all aggregate samples. All materials were used as obtained, without any further purification.

Preparation and absorption of aggregate solutions: Appropriate amounts of the dyes were weighed and dissolved in methanol to make monomer stock solutions, then diluted in methanol to make a series of monomer solutions of appropriate concentrations. Each diluted monomer solution was injected into water or aqueous $\mathrm{NaCl}$ with a specified mixing ratio. The concentration of the diluted monomer solution was decided such that the final concentration after water/ aq. $\mathrm{NaCl}$ injection is held constant. The calculations for this process were streamlined in a MATLAB code (available upon request). Glass vials used to store the samples were pre-soaked in Milli- $Q$ water and aggregate solutions were allowed to equilibrate in dark for $24 \mathrm{hrs}$ prior to measurement. Absorption measurements were taken on Agilent Cary 60 spectrometer for visible/NIR samples. SWIR absorption spectra were taken on JASCO V-770 UV/Vis/NIR 
spectrometer. Starna Cells quartz cuvettes of path lengths $0.01 \mathrm{~mm}, 0.2 \mathrm{~mm}$ or $1 \mathrm{~mm}$ were used depending on the dye concentration.

Cryo-electron microscopy (CryoEM): Samples were prepared on mesh 200 lacey formvar/carbon copper grids obtained from Ted Pella Inc. The grids were plasma-cleaned under a $\mathrm{H}_{2} / \mathrm{O}_{2}$ gas flow using a Solarus Gatan Plasma cleaner for hydrophilization. Vitrobot Mark IV was used for plunge-freezing the samples. 5 $\mu \mathrm{L}$ of the aggregate solution was dropped onto the grid, and excess solution was removed by blotting for $3.0 \mathrm{~s}$ with standard blotting paper from Ted Pella and immediately dropped into liquid ethane. The frozen grids were stored in liquid nitrogen. Grids were loaded on to a liquid nitrogen cooled Gatan 626 cryotransfer sample holder, then inserted into the microscope. CryoEM images were recorded on a FEI TF20 electron microscope equipped with a field-emission gun at $200 \mathrm{keV}$ and CCD camera.

Atomic force microscopy (AFM): Films for AFM were prepared according to a previously reported dropflow technique with some modifications. ${ }^{2}$ Aggregate solutions (2-3 drops) were dropped on a freshly cleaved mica substrate $(1 \mathrm{~cm}$ diameter) kept at a small incline. Mica substrates were obtained from Thermo Fisher Scientific. The solutions were allowed to slowly air dry on the bench while covered with a box to shield from room light and air currents. AFM measurements were done on Veeco Bioscope II AFM with tapping mode Al coated AFM tips obtained from NanoAndMore USA Corp. with $42 \mathrm{~N} / \mathrm{m}$ force constant and a resonant frequency of $\sim 320 \mathrm{kHz}$.

Diffusion ordered spectroscopy (DOSY) NMR: NMR spectra were taken at room temperature on a Bruker Avance $500 \mathrm{MHz}$ spectrometer with a $5 \mathrm{~mm}$ broadband probe and processed using TopSpin 4.07. After finding sufficient values for gradient pulse length $(p 30, \delta)$ and diffusion time $(d 20, \Delta)$ to yield spectra with 95 and $5 \%$ of original signal, 32 spectra were taken on a linear ramp and processed through TopSpin for diffusion constants.

Sugar matrix stabilization: Prepared solutions of 2D aggregates after the $24 \mathrm{hr}$ equilibration step were mixed with a saturated sugar solution made by dissolving a 50:50 sucrose/trehalose $(\mathrm{w} / \mathrm{w})$ in distilled water. $100 \mu \mathrm{L}$ aggregate solution was taken in a vial pre-soaked in Milli-Q water. To this, $100 \mu \mathrm{L}$ of the sugar solution was added drop-wise and gently mixed. The sugar-aggregate mixture was dropped onto a $0.2 \mathrm{~mm}$ quartz cuvette from Starna Cells Inc. and kept under vacuum in dark for $24 \mathrm{~h}$ for drying.

\section{References}

(1) Deshmukh, A. P.; Koppel, D.; Chuang, C.; Cadena, D. M.; Cao, J.; Caram, J. R. Design Principles for Two-Dimensional Molecular Aggregates Using Kasha's Model: Tunable Photophysics in Near and Short-Wave Infrared. J. Phys. Chem. C 2019, 123 (30), 18702-18710.

https://doi.org/10.1021/acs.jpcc.9b05060.

(2) Eisele, D. M.; Knoester, J.; Kirstein, S.; Rabe, J. P.; Vanden Bout, D. A. Uniform Exciton Fluorescence from Individual Molecular Nanotubes Immobilized on Solid Substrates. Nat. Nanotechnol. 2009, 4 (10), 658-663. https://doi.org/10.1038/nnano.2009.227.

Notes 'N. S. (https://math.stackexchange.com/users/9176/n-s), Is there an analytical way to find the number of real positive roots of polynomials of the form $\mathrm{ax}^{\wedge} \mathrm{m}+\mathrm{bx}^{\wedge} 2+\mathrm{x}-\mathrm{c}=0$ ?, URL (version: 2019-12-20):

https://math.stackexchange.com/q/3483221. 